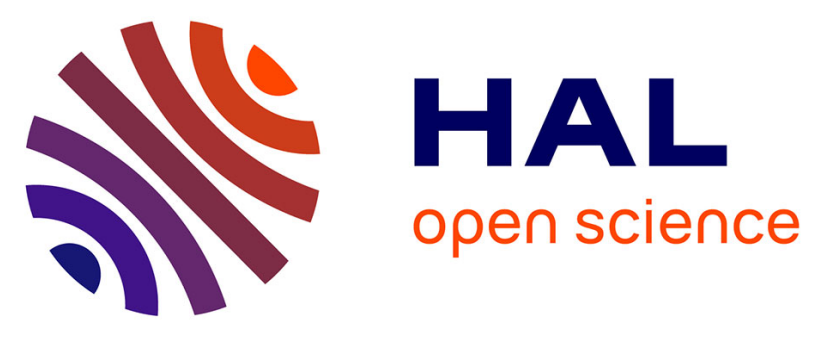

\title{
Electric field-assisted metal insulator transition in vanadium dioxide (VO2) thin films: optical switching behavior and anomalous far-infrared emissivity variation
}

Aurelian Crunteanu, Fabert Marc, Julie Cornette, Maggy Colas, Jean-Christophe Orlianges, Annie Bessaudou, Françoise Cosset

\section{To cite this version:}

Aurelian Crunteanu, Fabert Marc, Julie Cornette, Maggy Colas, Jean-Christophe Orlianges, et al.. Electric field-assisted metal insulator transition in vanadium dioxide (VO2) thin films: optical switching behavior and anomalous far-infrared emissivity variation. Proceedings SPIE 9364, Oxide-based Materials and Devices VI , 2015, Proc. SPIE 9364, Oxide-based Materials and Devices VI, 9364, pp.93640J1-J11. 10.1117/12.2076260 . hal-01155619

\section{HAL Id: hal-01155619 \\ https://hal-unilim.archives-ouvertes.fr/hal-01155619}

Submitted on 27 May 2015

HAL is a multi-disciplinary open access archive for the deposit and dissemination of scientific research documents, whether they are published or not. The documents may come from teaching and research institutions in France or abroad, or from public or private research centers.
L'archive ouverte pluridisciplinaire HAL, est destinée au dépôt et à la diffusion de documents scientifiques de niveau recherche, publiés ou non, émanant des établissements d'enseignement et de recherche français ou étrangers, des laboratoires publics ou privés.

$$
\text { Copyright }
$$




\title{
Electric field-assisted metal insulator transition in vanadium dioxide (VO2) thin films: optical switching behavior and anomalous far-infrared emissivity variation
}

\author{
Aurelian Crunteanu* ${ }^{\mathrm{a}}$, Marc Fabert ${ }^{\mathrm{a}}$, Julie Cornette ${ }^{\mathrm{b}}$, Maggy Colas $^{\mathrm{b}}$, Jean-Christophe Orlianges ${ }^{\mathrm{b}}$, \\ Annie Bessaudou ${ }^{\mathrm{a}}$, Françoise Cosset ${ }^{\mathrm{a}}$ \\ ${ }^{a}$ XLIM UMR 7252 - CNRS/ University of Limoges- 123 avenue Albert Thomas - 87060, Limoges; \\ France; ${ }^{\mathrm{b}}$ SPCTS UMR 7315- CNRS/ University of Limoges- 12 rue Atlantis, 87068, Limoges, \\ France
}

\begin{abstract}
We present the vanadium dioxide $\left(\mathrm{VO}_{2}\right)$ thin films deposition using e-beam evaporation of a vanadium target under oxygen atmosphere on different substrates (sapphire, $\mathrm{Si}, \mathrm{SiO}_{2} / \mathrm{Si} \ldots$ ) and we focus on their electrical and optical properties variations as the material undergoes a metal-insulator transition under thermal and electrical stimuli. The phase transition induces extremely abrupt changes in the electronic and optical properties of the material: the electrical resistivity increases up to 5 orders of magnitude while the optical properties (transmission, reflection, refractive index) are drastically modified. We present the integration of these films in simple planar optical devices and we demonstrate electrical-activated optical modulators for visible-infrared signals with high discrimination between the two states. We will highlight a peculiar behavior of the $\mathrm{VO}_{2}$ material in the infrared and far infrared regions $(2-20 \mu \mathrm{m})$, namely its anomalous emissivity change under thermal- end electrical activation (negative differential emittance phenomenon) with potential applications in active coatings for thermal regulation, optical limiting or camouflage coatings.
\end{abstract}

Keywords: vanadium dioxide, metal-insulator transition, electrical and optical properties, optical modulation, thermal emissivity variation

\section{INTRODUCTION}

Vanadium dioxide (VO2) is an electron-correlated material performing a first-order metal-insulator phase transition (MIT) characterized by a reversible, temperature-driven structural phase change near $\mathrm{T}_{\mathrm{MIT}}=68^{\circ} \mathrm{C}^{1-4}$. The material evolves from a monoclinic-type, insulating state (below $\mathrm{T}_{\mathrm{MIT}}$ ) to a tetragonal structure with metallic properties (above $\mathrm{T}_{\mathrm{MIT}}$ ). The electrical and optical properties of $\mathrm{VO}_{2}$ films and nanostructures are drastically modified between these two states: thus, their electrical resistivity can vary by up to $4-5$ orders of magnitude while the optical transmission and the refractive index are largely modified. Beside the temperature activation, the $\mathrm{MIT}$ in $\mathrm{VO}_{2}$ thin films can be induced also electrically (by charge injection or Joule heating), optically (photon injection) or even by applying high pressure or stress to the material ${ }^{4-9}$. The optical- and electrical-field induced transitions are expected and even reported to take place very fast, under $1 \mathrm{ps}^{9}$. These properties are potentially very interesting for fast electrical and optical switching and modulation, making vanadium dioxide a promising material for applications in oxide-based adaptive electronics and optics or sensing devices $^{8,10-13}$. Due to its relative low transition temperature and its rich electro-optical non-linear properties within the MIT phase transition, $\mathrm{VO}_{2}$ become an archetypal system for studying the physics behind the metal-insulator transition ${ }^{1-4,}$ 9,14. However, the MIT mechanism in VO2 is still matter to debate, opposing a temperature-driven structural process, related to electron-phonon interactions (Peierls transition - SPT) ${ }^{4,9}$ to a pure electronic mechanism based on electronelectron correlations or Mott transitions ${ }^{2,7}$. In this paper we present the general optical and electrical properties of $\mathrm{VO}_{2}$ thin films performing a thermally- or electrically-triggered MIT and we focus on their unusual non-linear optical properties in the infrared and far-infrared regions of the optical spectrum. We will show the realization of optical modulators using the electrical activation of the MIT in the $\mathrm{VO}_{2}$ layers and we will analyze their dynamic operation characteristics, highlighting the modulation frequency limits and specific functioning regimes.

*aurelian.crunteanu@xlim.fr; phone +33 5.87.50.67.41; fax +33 5.55.45.76.49; www.xlim.fr 


\section{EXPERIMENTAL}

Over the time, various deposition techniques have been employed to grow high quality $\mathrm{VO}_{2}$ thin films, such as pulsed laser deposition, reactive ion beam sputtering or magnetron sputtering ${ }^{15-17}$. Here, the $\mathrm{VO}_{2}$ coatings were deposited by electron-beam evaporation of a vanadium target under pure oxygen atmosphere in the presence of a radiofrequency electrical discharge, on different substrates (sapphire, oxidized silicon and fused silica) ${ }^{18}$.

Typical experimental deposition parameters values for obtaining VO2 films with large differences in their electrical and optical properties across the MIT phase transition (when changing from insulating to metallic state) are: substrate temperature- $450-550^{\circ} \mathrm{C}$, oxygen pressure- $0.06-0.1 \mathrm{~Pa}$, RF incident power applied to the substrates- $10-50 \mathrm{~W}$ and deposition rates between 0.03 and $0.07 \mathrm{~nm} / \mathrm{s}$. Following film deposition, the temperature-dependent structural, electrical and optical properties of the experimentally optimized VO2 layers deposited on three different substrates (sapphire, thermally oxidized silicon $-1-\mu \mathrm{m}$ thick $\mathrm{SiO} 2 / \mathrm{Si}$ and fused silica) were investigated using X-ray diffraction (XRD), four-points electrical resistivity measurements, Raman spectroscopy, UV-vis (200-1600 nm) and FTIR spectroscopy (2 $\mathrm{mm}-24 \mathrm{~mm}$ ). The resistivity and optical transmission data of the obtained films were recorded during heating/cooling cycles (between $25^{\circ}$ and $95^{\circ} \mathrm{C}$ ) by using a current-regulated thermoelectric element (Peltier) with a 3- mm diameter traversing hole in its center. The fabricated films were integrated in simple electrical devices (planar two-terminal $\mathrm{VO}_{2^{-}}$ based switches) in order to evaluate the electrical activation of MIT in the material and its impact on the optical properties of the layers. The devices were realized in a clean room environment using classical micro-fabrication techniques $\left(\mathrm{VO}_{2}\right.$ patterning using wet etching and metallic electrodes fabrication using optical lithography and e-beam deposition $)^{19}$. The current-voltage (I-V) characteristics of the fabricated switches were recorded using a simple electrical circuit including the $\mathrm{VO}_{2}$ device in series with a resistance and a source meter (Keithley 2612A). The voltages across the $\mathrm{VO} 2$ switch and of the series resistance were recorded using a large bandwidth oscilloscope (Tektronik DPO7254).

\section{RESULTS AND DISCUSSIONS}

The $\mathrm{VO}_{2}$ films obtained by the electron beam evaporation technique are smooth (rms roughness in the order of 1-3 nm) and highly uniform. Their thickness variation, when fabricated on sapphire substrates with surfaces as large as two square inches, remains below 5\% (as revealed by mapping spectroscopic ellipsometry measurements, not shown here). Figure 1 shows the images of typical $\mathrm{VO}_{2}$ layers having different thickness, obtained on square, 20x20 mm sapphire substrates. As observed, the films color is progressively changing with from green-yellow (for $15-\mathrm{nm}$ thick films) to brownish for a thickness equal or superior to $100 \mathrm{~nm}$. The XRD analysis of the obtained films on sapphire substrates revealed that, independent of their thickness, all films correspond to the $\mathrm{VO}_{2}$ phase with a single (010) orientation with respect to the underlying (001) sapphire substrate. Moreover, grazing incidence diffraction (scanning $2 \theta$ at a fixed $\omega$ incidence) evidenced the absence of any disoriented phase. However, the XRD spectra of $\mathrm{VO}_{2}$ films obtained on $\mathrm{Si}$ or $\mathrm{SiO}_{2}$ substrates show multiple diffraction peaks, corresponding to the $\mathrm{M} 1$ phase of $\mathrm{VO} 2$ and reveal the polycrystalline nature of these deposits.

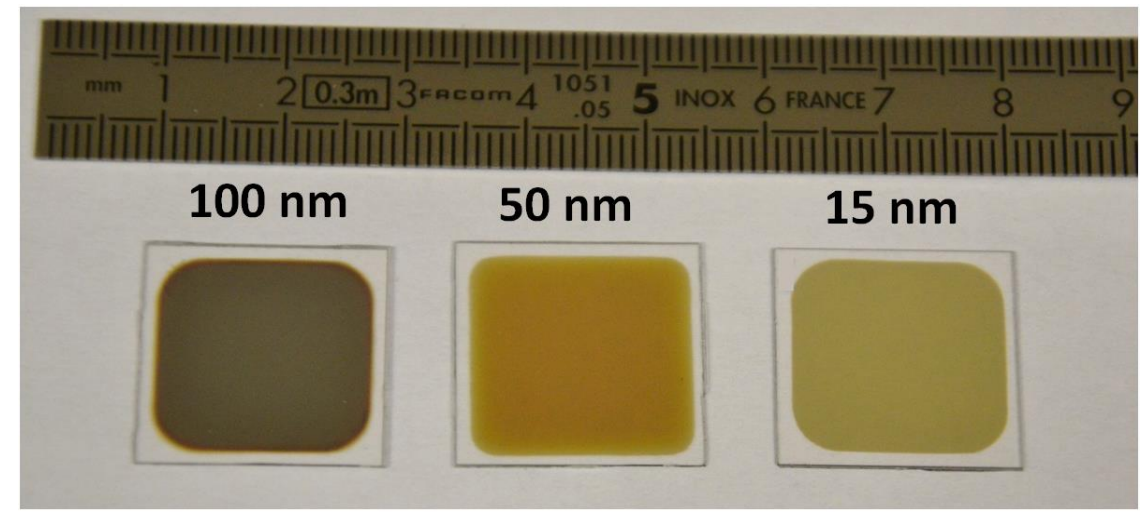

Figure 1. Photographs of typical VO2 films with different thickness obtained by reactive electron-beam evaporation on 20x20 mm2 sapphire substrates. 


\subsection{Electrical and optical properties of the fabricated $\mathrm{VO}_{2}$ layers}

The four-point electrical resistivity of the layers obtained on different substrates was recorded while applying heating/ cooling temperature cycles in the $25^{\circ} \mathrm{C}-100^{\circ} \mathrm{C}$ interval. Figure 2 shows the temperature-dependent hysteresis of the normalized resistivity for 100-nm thick VO2 layers obtained on three different substrates: sapphire, silicon ( $\mathrm{Si}$ ) and fused silica ( $\mathrm{SiO} 2)$. The normalized resistivity of the films is defined as the ratio $\rho \mathrm{i} / \rho 0$ with $\rho 0$ the resistivity in the metallic state of the films (at $95{ }^{\circ} \mathrm{C}$ ) and $\rho$ i the electrical resistivity measured in the range $25^{\circ} \mathrm{C}-95^{\circ} \mathrm{C}$. For all types of VO2 deposits, one may observe a drastic change of the normalized resistivity, by several orders of magnitude, as the samples temperatures pass across the MIT transition temperature. The highest resistivity change (magnitude of the MIT) are obtained for the films obtained on sapphire, which shows a resistivity change of up to five orders of magnitude compared with the three orders of magnitude resistivity change on silicon and silica substrates. The transition temperatures $\left(\mathrm{T}_{\mathrm{MIT}}\right)$ and the resistivity hysteresis widths $(\Delta \mathrm{T})$ vary also with the type of the substrate. Thus, for sapphire $\mathrm{T}_{\mathrm{MIT}}$ is around $68.9^{\circ} \mathrm{C}$ with $\Delta \mathrm{T} \sim 3.7^{\circ} \mathrm{C}$ while for $\mathrm{SiO}_{2}$ substrates, $\mathrm{T}_{\mathrm{MIT}} \sim 66^{\circ} \mathrm{C}$ and $\Delta \mathrm{T} \sim 6.1^{\circ} \mathrm{C}$. A large magnitude of the resistivity change during MIT is usually accompanied by a small resistivity hysteresis width. The variation of $\mathrm{T}_{\mathrm{MIT}}$ and $\Delta \mathrm{T}$ for the different $\mathrm{VO}_{2}$ films indicates the influence of the substrates or of the underlying layers on the film MIT properties (modification of their structure/ degree of crystallization, apparition of defects and grain size effects) $)^{20}$.

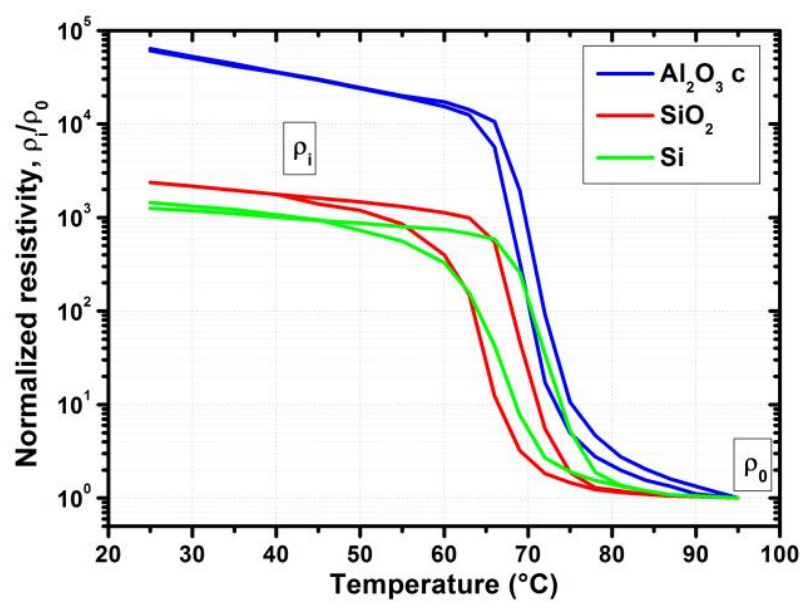

Figure 2. Typical electrical resistivity hysteresis curves (resistivity records during heating/cooling cycles) of VO2 samples obtained on different substrates.

In order to characterize the optical properties of the $\mathrm{VO}_{2}$ films obtained on sapphire substrates across the MIT, we recorded their optical transmission curves in the UV-vis- IR regions of the spectrum at different temperatures, in the 20$85^{\circ} \mathrm{C}$ range, similarly, during consecutive heating/ cooling cycles. On Figure 3 is represented the temperature- dependent variation (between $20^{\circ}$ and $85^{\circ} \mathrm{C}$ )) of the optical transmission of a $100-\mathrm{nm}$ thick $\mathrm{VO}_{2}$ layer, in the $200 \mathrm{~nm}-1600 \mathrm{~nm}$ spectral domain, for heating -Figure $3 \mathrm{a}$, and cooling cycles -Figure 3b, respectively.

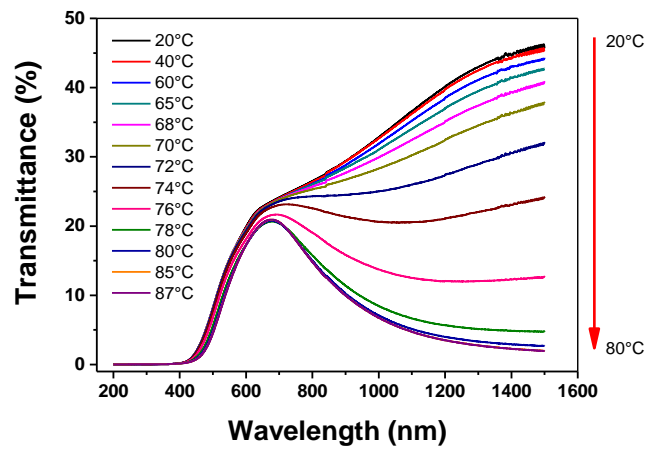

a.

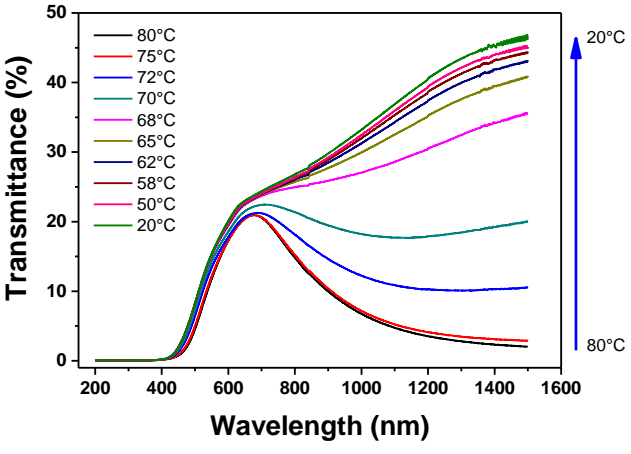

b.

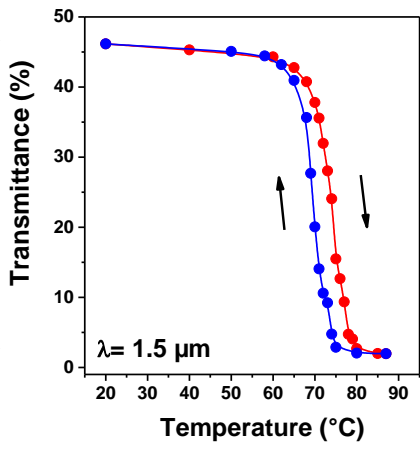

c.

Figure 3. Temperature dependence of the optical transmission from 200 to $1600 \mathrm{~nm}$ for a 100-nm thick VO2 films on sapphire: a. transmission variation for different temperatures during the heating cycle, $b$. the same recordings during the 
cooling cycle and $\mathrm{c}$. the hysteresis cycle at $\lambda=1500 \mathrm{~nm}$ inferred from figures a and $\mathrm{b}$, showing a sharp transmittance variation associated with the MIT in the VO2 thin film.

One may notice that the optical transmission is strongly changing across the temperature-induced MIT, especially for the longer wavelengths (in the IR region of the spectral domain) ${ }^{18}$. Similar behaviour was noticed for $\mathrm{VO}_{2}$ films obtained on other transparent substrates $\left(\mathrm{SiO}_{2}\right)$, although the ratio change of the transmittance (at $20^{\circ} \mathrm{C}$ and at $\left.80^{\circ} \mathrm{C}\right)$ is much lower than for the films obtained on sapphire substrates (for which the transmission can change by a factor of $\sim 500$ around 1.5 $\mu \mathrm{m}$ when heating the sample in the same temperature interval). Using the data presented on Figures $3 \mathrm{a}$ and $3 \mathrm{~b}$, we represented on Figure $3 \mathrm{c}$ the thermal hysteresis of the transmittance at $\lambda=1500 \mathrm{~nm}$. As observed, the optical transmission of the $\mathrm{VO}_{2}$ film on sapphire show a sharp profile and a very low hysteresis width, witnessing of abrupt changes in the film's optical properties across the metal-insulator transition.

\subsection{Far-Infrared properties and negative differential thermal emittance phenomena of the $\mathrm{VO}_{2} /$ sapphire system $^{2}$}

Following the optical characterization of the $\mathrm{VO}_{2}$ layers in the UV-visible domain, we investigated their properties on a larger spectral domain, in the mid- and far infrared (2- $24 \mu \mathrm{m})$, using the Fourier-Transformed Infrared spectroscopy. It was recently demonstrated ${ }^{21}$ that the system comprising a thin $\mathrm{VO}_{2}$ layer on a sapphire substrate behave like a "natural disordered metamaterial" and shows perfect absorption and large tuning ranges, at specific IR wavelengths (11-12 $\mu \mathrm{m})^{22}$. We investigated using FTIR the temperature-dependent reflectivity of similar systems made of a $\mathrm{VO}_{2}$ layer (having thickness between 50 and $200 \mathrm{~nm}$ ) obtained on a sapphire substrate. On Figure 4 are represented the IR reflectivity at different temperatures (between $30^{\circ} \mathrm{C}$ and $80^{\circ} \mathrm{C}$ ) for a $50-\mathrm{nm}$ thick $\mathrm{VO}_{2}$ layer on a sapphire substrate. If we focus on the reflectivity values in the $10.5-12.5 \mu \mathrm{m}$ spectral interval (highlighted in dotted lines on Figure 4), we may notice that for temperatures between $30^{\circ} \mathrm{C}$ and $48^{\circ} \mathrm{C}\left(\mathrm{VO}_{2}\right.$ layer in the insulating state and transparent in the IR), the reflectivity is quite high, around $45-52 \%$ and mainly coming from the underlying sapphire substrate. For temperatures higher than $60^{\circ} \mathrm{C}$, the $\mathrm{VO}_{2}$ film become metallic and the overall reflectivity of the system is close to $40 \%$ (mostly coming from the highreflective $\mathrm{VO}_{2}$ layer). However, for intermediate temperatures $\left(50\right.$ to $\left.60^{\circ} \mathrm{C}\right)$, corresponding to the onset of the metalinsulator transition in the $\mathrm{VO}_{2}$ layer, the reflectivity is drastically reduced to below $5 \%$ (for temperatures at the beginning of the MIT) and is further increasing until the material reach its metallic state. As mentioned earlier ${ }^{21}$, the absorption depth and its spectral position depend on $\mathrm{VO}_{2}$ layer thickness. We verified the same phenomena on different other layersubstrate systems and different activation configurations (pure and doped- or structured $\mathrm{VO}_{2}$ layers on sapphire or $\mathrm{Si}$ substrates, thermal or electrical activation, results not reported here).

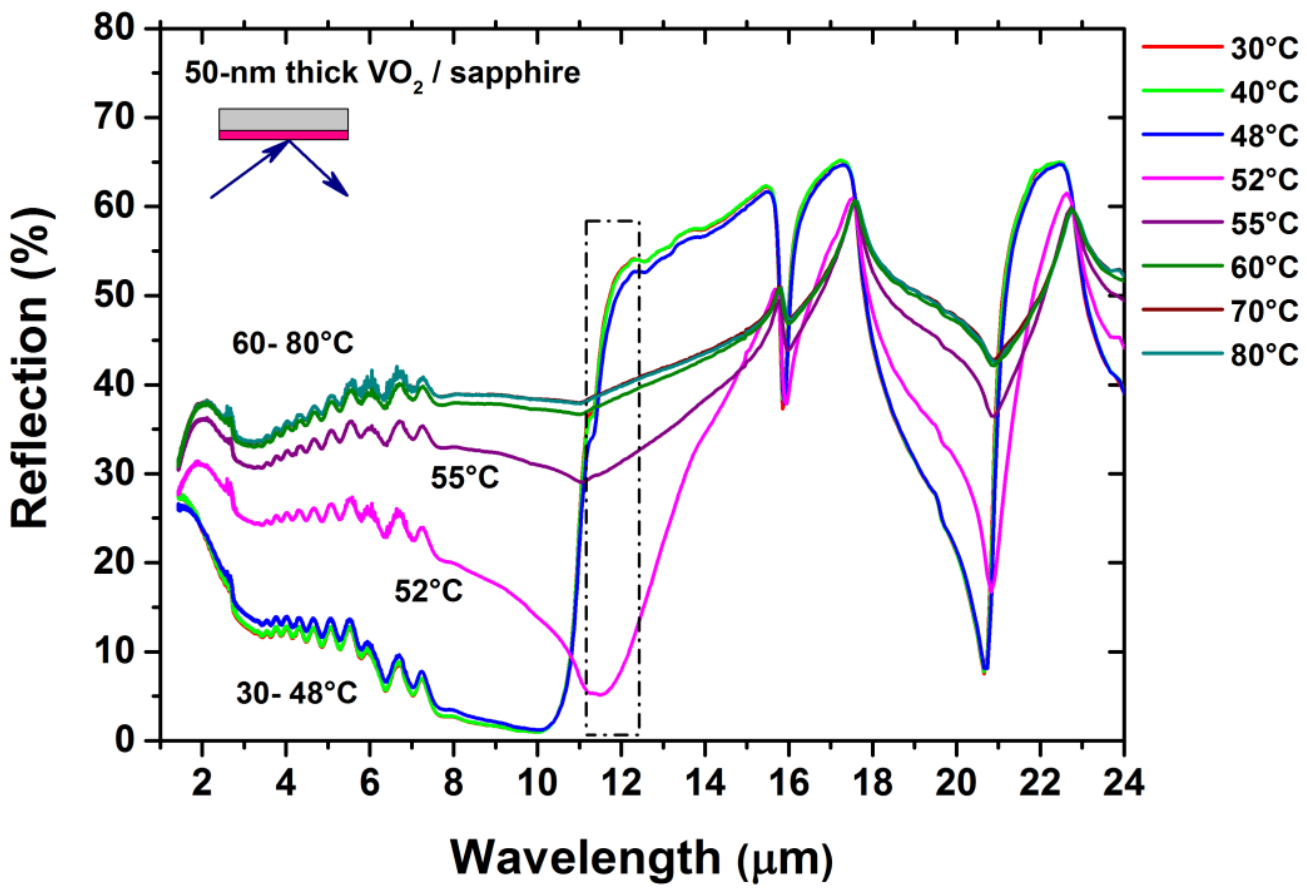

Figure 4. Temperature-dependent reflectivity curves for a 50-nm thick VO2 layer on a sapphire substrate, obtained by FTIR spectroscopy. 
The results presented before suggest that the $\mathrm{VO}_{2}$ layers on sapphire behave like a thermochromics system presenting a negative differential reflectivity and negative differential thermal emittance through the variation of their optical constants during the metal-insulator transition of the $\mathrm{VO}_{2}$ layer ${ }^{22}$. These phenomena can be easily and intuitively illustrated by imaging a $\mathrm{VO}_{2}$ layer heated across its transition temperature, using a thermal camera (Fluke Ti10 Thermal Imager). In Figure 5 are presented such thermal images taken during the heating of a 100 -nm thick $\mathrm{VO}_{2}$ layer on a $50 \mathrm{x} 50$ $\mathrm{mm}^{2}$ sapphire substrate (placed above a Peltier heating element of $20 \times 20 \mathrm{~mm}^{2}$ ). It may be clearly observed that while heating, the sample display negative differential thermal emittance above the MIT transition temperature (around $70^{\circ} \mathrm{C}$ ): the sample appears cooler even if the applied temperature is increasing above the $\mathrm{T}_{\text {MIT }}$.

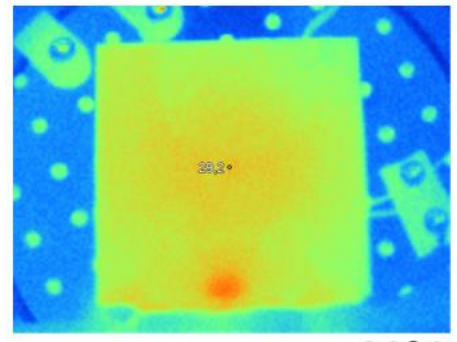

$30^{\circ} \mathrm{C}$

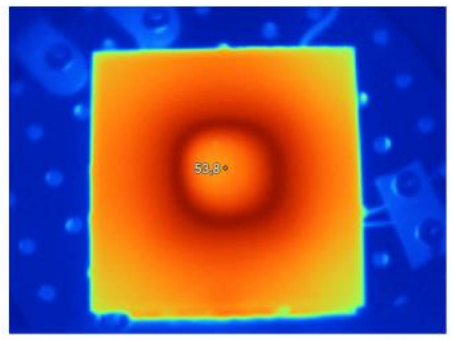

$75^{\circ} \mathrm{C}$

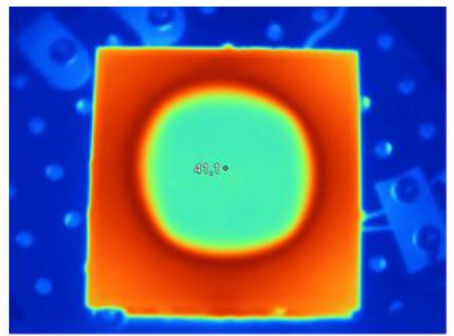

$85^{\circ} \mathrm{C}$

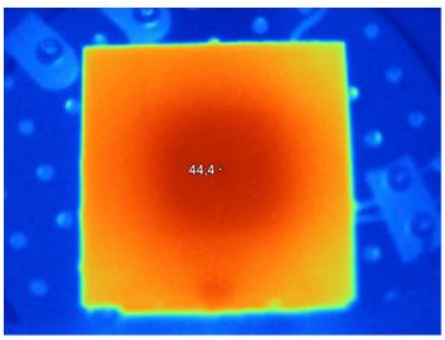

$50^{\circ} \mathrm{C}$

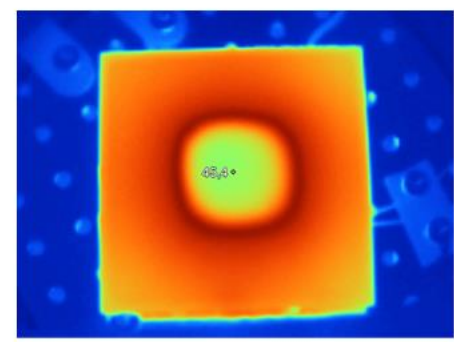

$77^{\circ} \mathrm{C}$

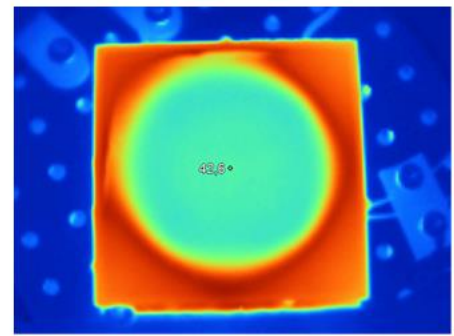

$90^{\circ} \mathrm{C}$

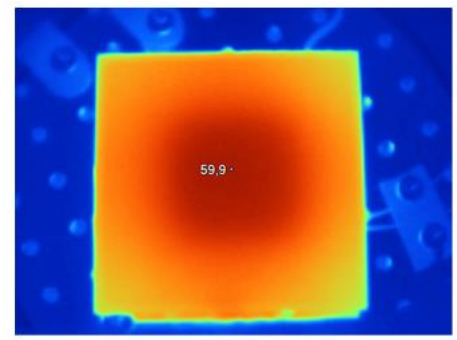

$65^{\circ} \mathrm{C}$

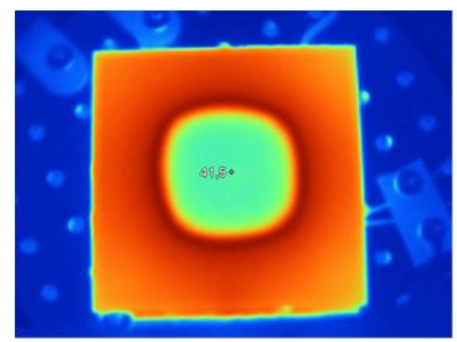

$80^{\circ} \mathrm{C}$

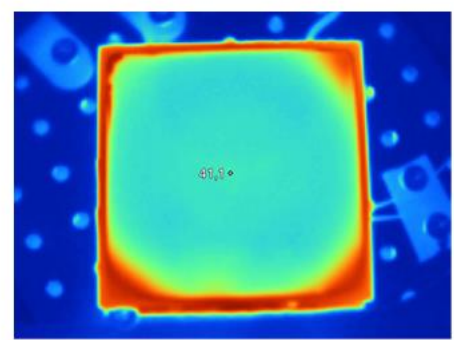

$100^{\circ} \mathrm{C}$

Figure 5. IR thermal images of a $50 \times 50 \mathrm{~mm}^{2}$ square 100 -nm thick $\mathrm{VO}_{2}$ layer on a sapphire substrate showing the phenomenon of negative differential emittance while heated across its MIT transition temperature.

We demonstrated the same phenomenon by using combined thermal- and electrical- activated MIT and investigated different other films-substrate combinations, including structured VO2 films. In future reports (under preparation) we will show that it is possible to use the VO2 material as a medium which may store or encode information that can only be retrieved in particular conditions (temperature range, electrical bias) at these specific IR wavelengths.

\subsection{Optical modulation using the electrically- activated $\mathrm{MIT}$ in $\mathrm{VO}_{2}$ thin films}

The electrical activation of MIT (E-MIT) was intensively studied in $\mathrm{VO}_{2}$ films ${ }^{7,8,19,23}$ since it may occur on faster timescales and offer a higher degree of integration compared to the temperature-induced MIT. For evaluating the electricallyinduced optical switching capabilities of the $\mathrm{VO}_{2}$ thin films obtained on sapphire substrates, we fabricated two-terminal type (2T) devices (two contact metallic electrodes deposited apart on $\mathrm{VO}_{2}$ film patterns). Figure 6 shows images of typical planar $2 \mathrm{~T} \mathrm{VO}_{2}$-based switches (fabricated in a clean-room environment) for which we studied the influence of their length and width (distance and width of the two opposite electrodes) on their current-voltage (I-V) characteristics. 

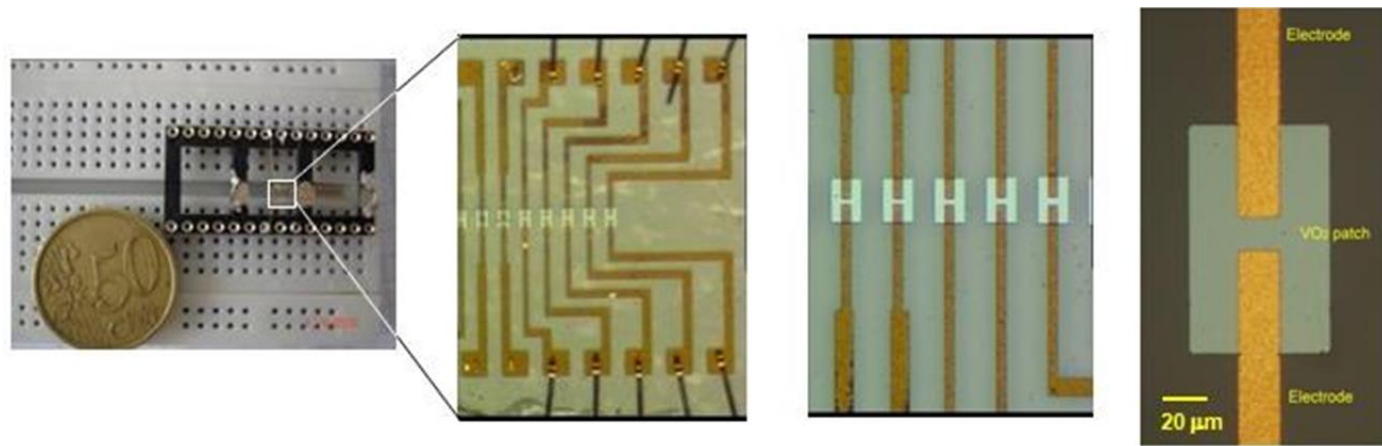

Figure 6. Photographs of typical fabricated two-terminal devices integrating $\mathrm{VO}_{2}$ patterns obtained on sapphire substrates.

The I-V curves of a 20- $\mu$ m length VO2 device are presented on Figure 7a, for two different actuation modes: V-mode and respectively I-mode (imposing a voltage sweep while recording the current in the circuit for the V-mode or imposing a current-sweep and recording the voltage drop across the VO2 device for the I-mode, respectively). The simple electrical set-up used for these recordings is presented in the inset of Figure 7a. The I-V curve recorded in the V-mode shows that the $\mathrm{VO}_{2}$ material undergoes a clear transition (E-MIT) from a high resistance insulating state to a low resistance metallic one, at a typical threshold voltage ( $\mathrm{Vth}=24 \mathrm{~V}$ for the specific device under test (DUT) having a 20$\mu \mathrm{m}$ length). We may notice a marked electrical hysteresis of the I-V characteristic, which can be explained by thermal effects associated with Joule heating due to high values of the currents after the insulator-to-metal transition. The threshold voltage values are lowering with decreasing the distance between the two electrodes (device length) and can reach values as low as several volts for device lengths of several microns or below ${ }^{6,23}$. Compared with the V-mode, the current-voltage characteristic in the I-mode activation of the $\mathrm{VO}_{2}$ device shows a similar E-MIT behavior: a sudden current jump between the two extreme states of the material, at similar voltage threshold values.
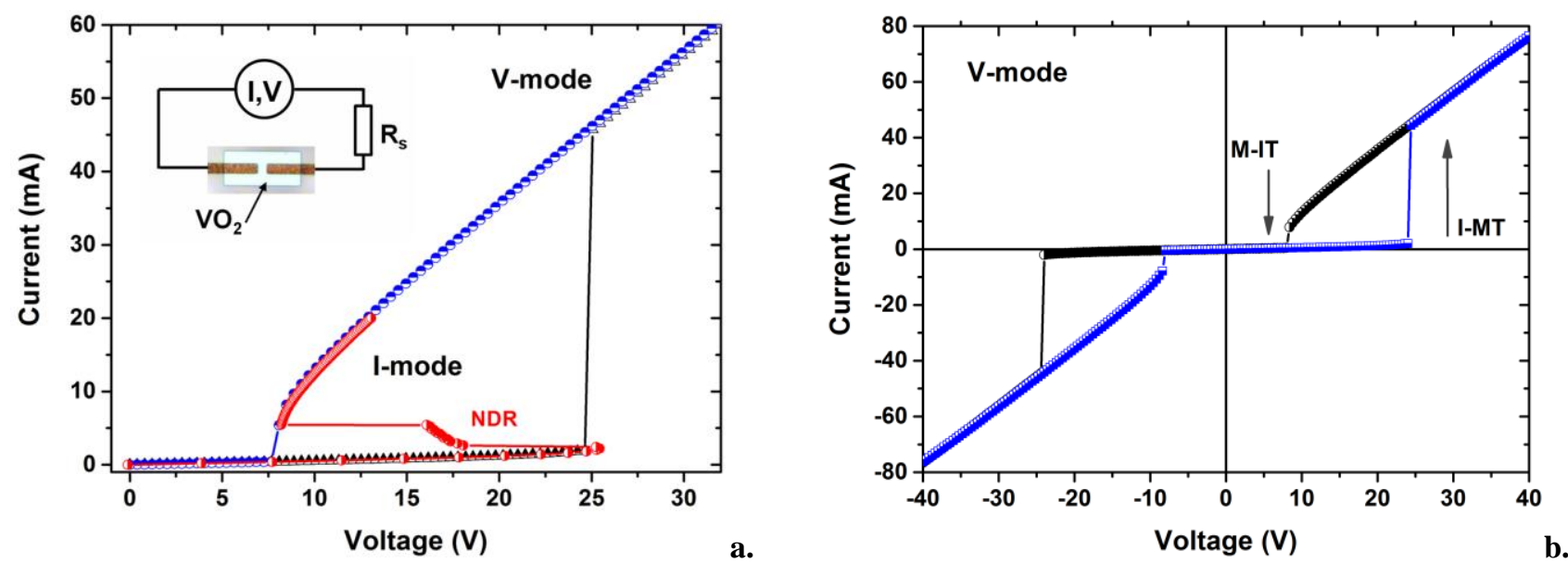

Figure 7. a. I-V characteristic in both V-and I-modes of a device incorporating a 20- $\mu$ m length VO2 pattern, with in insert, the electrical testing circuit; $b$. Current-voltage curves for the same device during positively and negatively sweeping of the applied voltage (V-mode operation) showing symmetrical behavior with respect to the origin of the I-V scale.

However one may notice a major difference for the I-mode operation, namely the progressive decrease of the voltage across the DUT with increasing current, after the MIT activation in $\mathrm{VO}_{2}$ at $\mathrm{V}$ th. This intermediate region in the I-V curve recorded in the I-mode is corresponding to a negative differential resistance (NDR) over the device. As previously discussed, the presence of the NDR regions in the I-V curves of $\mathrm{VO}_{2}$ devices activated in the current-mode can trigger self-sustained electrical oscillations across the device under constant current injection inside the NDR ${ }^{19,24}$. Such nonlinear electrical behavior may be used for realizing current-controlled inverters and nano-scale oscillators for applications in high-speed, large scale integrated circuits but can also offer some insights to the understanding of the driving mechanism in such metal-insulator devices.

The I-V curves of the VO2 devices (in both I-mode and V-mode operation) are perfectly symmetrical around the origin 
of the I-V scale, as exemplified for the V-mode operation of the same device, on Figure $7 \mathrm{~b}$ (voltage positively and negatively swept between 0 and $40 \mathrm{~V}$ ).

Besides this rich electrical behavior, the onset of the E-MIT in the $\mathrm{VO}_{2}$ material is accompanied by clear changes in its optical properties. These changes may be noticed even in the visible domain, during the electrical actuation of the $\mathrm{VO}_{2}$ device. Thus, on Figure 8 are presented some close-up views of the optical reflectivity change for a typical $\mathrm{VO}_{2} 2 \mathrm{~T}$ device (on a sapphire substrate) while applying to its electrodes a periodical triangular-shape voltage waveform $((1 \mathrm{~Hz}$, 40- V amplitude). The sequential images are extracted from the associated movie (Video 1) and were recorded by a CCD camera mounted on an optical microscope operating in reflection-mode.
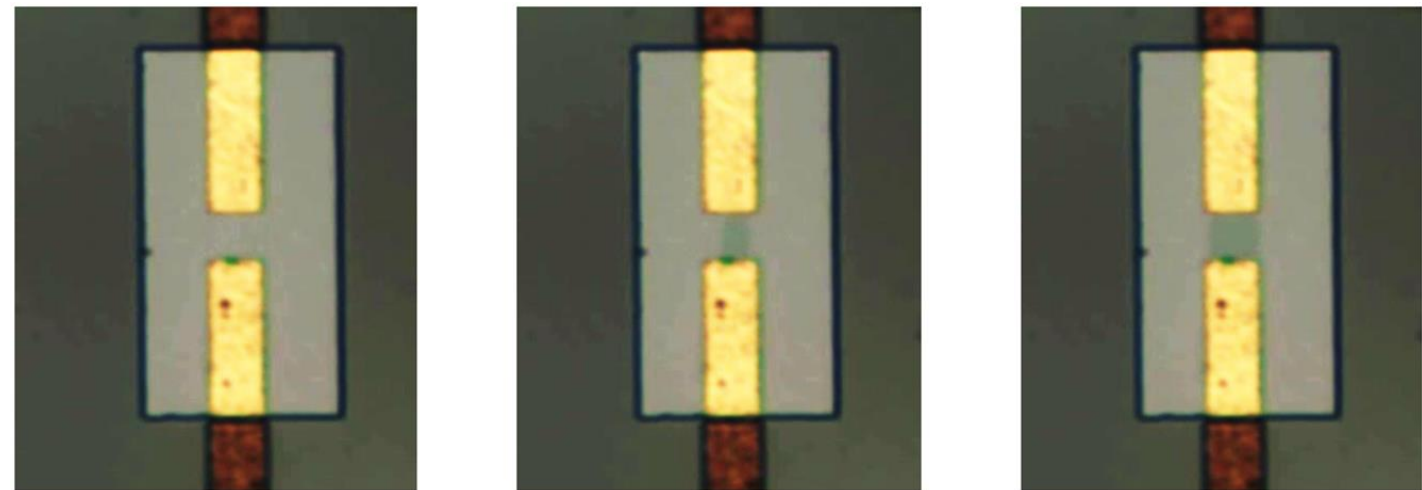

Figure 8. E-MIT inducing reflectivity changes of the VO2 material, recorded in reflection-mode optical microscopy while the applied voltage on the electrodes of the $2 \mathrm{~T}$ device is periodically swept between 0 and $40 \mathrm{~V}$.

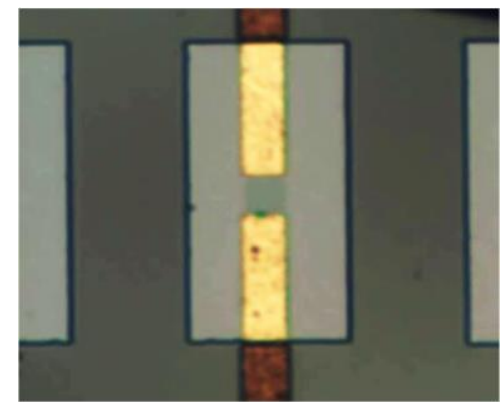

Video 1. Movie showing the periodical changes of the optical reflection of the $\mathrm{VO}_{2}$-based $2 \mathrm{~T}$ device during periodical onset of the E-MIT in the VO2 material (activation using a voltage waveform of $1 \mathrm{~Hz}$ and $40-\mathrm{V}$ amplitude).

http://dx.doi.org/doi.number.goes.here

The images presented on Figure 8 and the associated Video1 indicates the appearance of a metallic- $\mathrm{VO}_{2}$ filament during the voltage-controlled insulator-to-metal transition of the material. The filament appears at the E-MIT threshold voltage (Vth) and its width is increasing with further increase of the applied voltage (to values superior to Vth). As the applied voltage is lowered, the widths of the filaments decrease and disappear for voltage threshold corresponding to the reverse metal-insulator transition in the material. The metallic nature of these regions appearing during E-MIT was confirmed by Raman micro spectroscopy measurements (not presented here). Thus, the material evolves between two different optical states with dissimilar reflectivity (and transmission) properties, which is the pre-requisite for the realization of an optical switch or optical modulator device.

The dynamical optical behaviour of the device was recorded by placing the extremity (collimating lensed fibre with a mode field diameter of $\sim 16 \mu \mathrm{m}$ ) of an erbium doped fibre amplifier (EDFA, at $\lambda=1550 \mathrm{~nm}$ ) in the vicinity of the surface of the $\mathrm{VO}_{2}$ pattern within the $2 \mathrm{~T}$ electro-optical switch. The incident optical power at the surface of the sample has been limited to $100 \mu \mathrm{W}$ in order to avoid optically-induced thermal effects. The experimental set-up used to record the optical transmission variation of the device during its electrical activation is presented on Figure 9.

The optical power transmitted through the $\mathrm{VO}_{2}$ pattern shown on Figure 8 was recorded using a high-speed detector while a triangular-type voltage waveform was applied on its electrical contacts $(\sim 23 \mathrm{~V}$ amplitude, $1 \mathrm{~Hz}$ actuation 
frequency). The modulated transmission at $1550 \mathrm{~nm}$ of the optical switch (T) is shown on Figure10a (blue curve), along with the typical electrical parameters $\left(\mathrm{V}_{\mathrm{A}^{-}}\right.$applied voltage (black curve), and $\mathrm{V}_{\mathrm{VO}^{-}}$voltage drop across the $\mathrm{VO}_{2}$ DUT (red curve)). It may be observed that the transmitted optical signal is highly attenuated when the $\mathrm{VO}_{2}$ material change from the insulating to its metallic state.

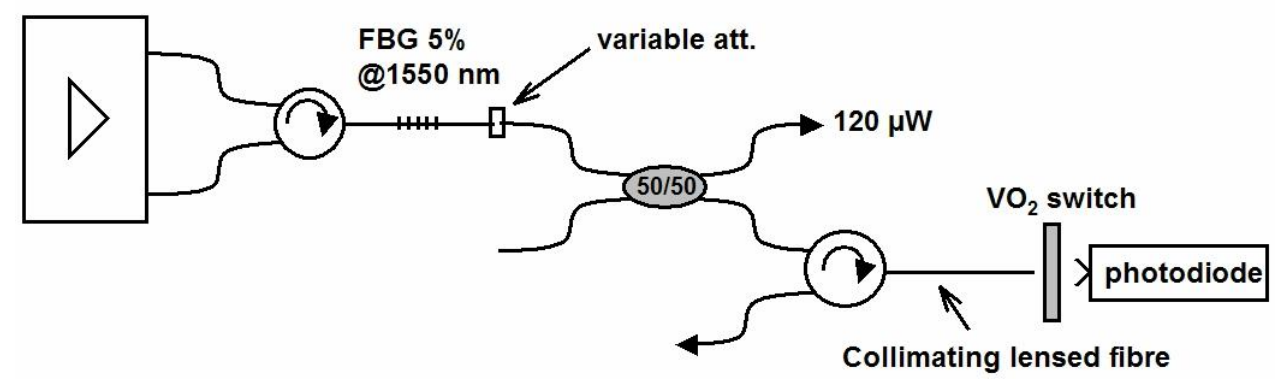

Figure 9. Experimental setup for the dynamical optical characterization (in transmission mode) of $\mathrm{VO}_{2}$ patterns on sapphire substrates during electrical activation of their MIT.

On Figure $10 \mathrm{~b}$ is presented the electrical hysteresis of the device (red curve) along with its optical transmission hysteresis (optical transmission response vs. the applied voltage during one period of the applied electrical signal). As in the case of the electrical hysteresis, the optical hysteresis widths is relatively large, implying strong thermal Joule effects associated with the E-MIT for this relatively large-sized device.
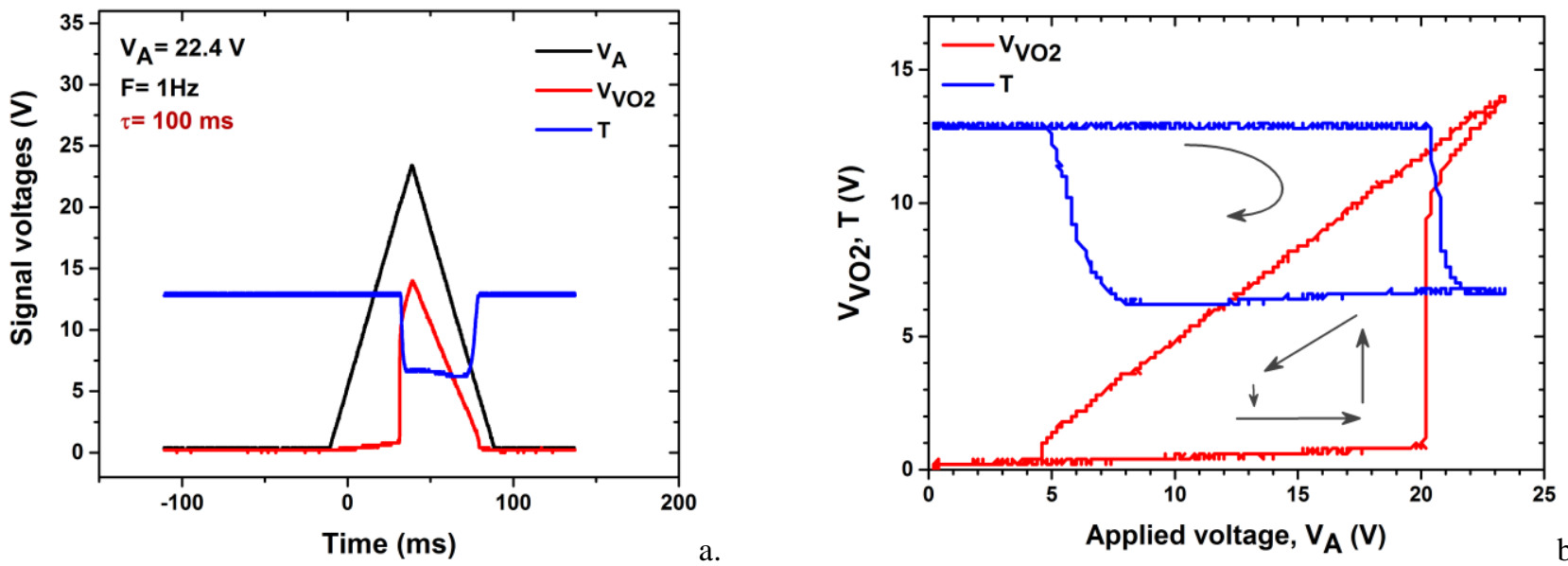

Figure 10. a. Dynamical optical transmission (at $1550 \mathrm{~nm}$ ) of electrically activated VO2 based optical device (black curve applied voltage, blue curve - voltage drop across the DUT, blue curve - optical transmission), and b. the hysteresis of the electrical response and of the optical transmission of the device.

The optical response time of a similar device was evaluated by recording the transmitted optical signal when applying square-type voltage waveforms of different widths, amplitudes and activation frequencies. A typical result of these studies is represented on Figure 11, which shows the transmission variation of the optical signal through the device (dT), during E-MIT activation using square-type voltage electrical pulses of 500- $\mu$ s length and different amplitudes (superior to the Vth of the device), at a fixed frequency, of $50 \mathrm{~Hz}$. A first observation referred from these curves is that the optical response of the device is decreasing with the increase of the applied voltage pulse amplitude but the response time still lies in the millisecond range. These values are much higher than the recorded electrical response of the switch (electrical resistivity change of the device recorded with the same type of voltage pulses), which typically lies around $200 \mathrm{~ns}$ for this type of device ${ }^{18,19}$. An explanation of this phenomenon can be related to the specificity of the optical set-up, in particular to the beam size of the light source incident on the $\mathrm{VO}_{2}$ pattern. Indeed, the progressive (and slow) expansion of the metallic phase at the surface of the sample (formation of the metallic filament) leads to a progressive fall in the transmitted optical power (or rise for the reflected power). The optical response may be further decreased at least by one 
factor of magnitude through an adapted design and a different activation scheme (imposed current or optical activation).

A second important observation is related to the optical delay recorded for the $\mathrm{VO}_{2}$ material to pass from the metallic state back to its initial, insulating state. Indeed, the metal-to insulator optical transition in the $\mathrm{VO}_{2}$ pattern seems to occur three to five times longer compared to the insulator-to metal transition, as previously reported for optical pump-probe experiments on plane $\mathrm{VO}_{2}$ layers 9 . The low-power transmitted optical signal (indicative of a metallic state of the VO2 pattern) is lasting even after removing the electrical activation pulses and suggests also the persistence of nonpercolative metallic domains within the $\mathrm{VO}_{2}$ material ("optical lag" with respect to the electrical response of the device. We noticed that for applied electrical signals with duty cycles (Dcy) inferiors to 5\%, the attenuation of the transmitted optical signal (1/dT) during the E-MIT and the "optical lag" delay are increasing with the electrical pulse amplitude (Figure 11) and the pulse width (not reported here). Under these conditions (Dcy<5\%), the actuation frequency does not have a noticeable influence on these parameters.

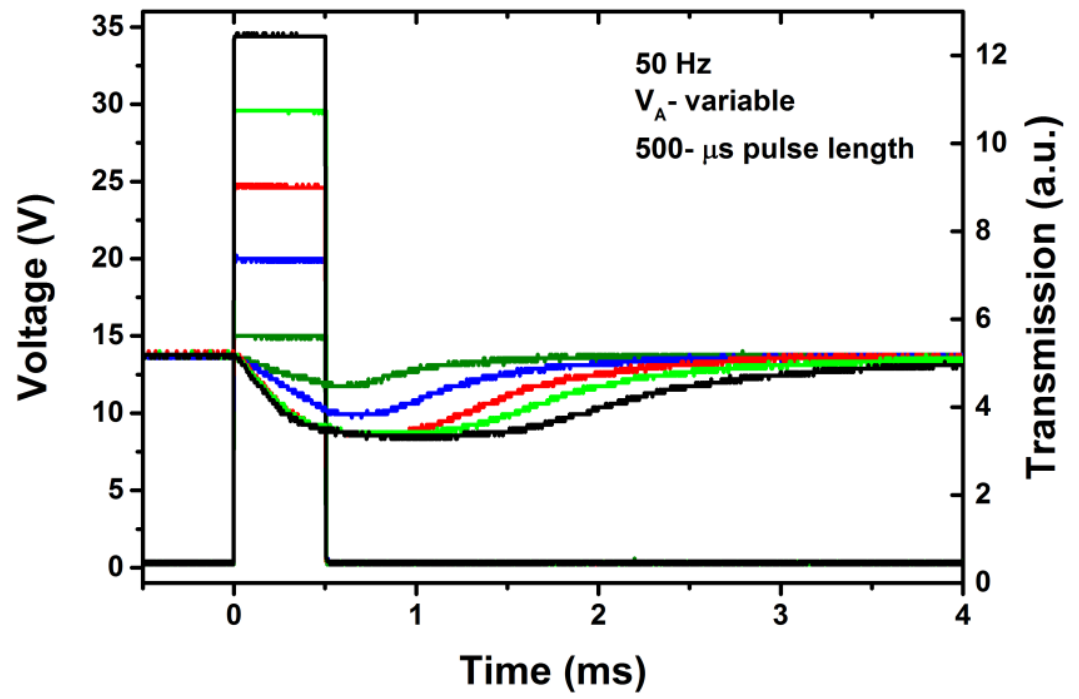

Figure 11. Temporal recordings of the optical transmission variation (dT) through the VO2 device during the E-MIT through the application of corresponding square-type voltage pulses with different amplitudes.

However, when applying electrical pulsed signals having Dcy> $10 \%$ to the optical $\mathrm{VO}_{2}$ device, the magnitude dT of the optical transmission variation is strongly dependent on the electrical signal frequency, as inferred from the graphs represented on Figure 12. As observed from the (a)-(f) red curves on Figure 12, the modulation of the optical signal transmitted through the device is decreasing with increasing the frequency of the electrical activation pulsed signals. For the typical devices we investigated (having lights between $15 \mu \mathrm{m}$ and $25 \mu \mathrm{m}$ ), the optical modulation depth is drastically decreasing at frequency values of several $\mathrm{kHz}$ (limit values depending of the device length, duty cycles or electrical pulse amplitude.

It is interesting to note that the presented device may be also perceived as an electrically-activated optical memory for which the variation of the transmitted optical power (dT) during the activation of MIT in the $\mathrm{VO}_{2}$ pattern plays the role of the state parameter of the device. Under specific and distinct activation schemes this state parameter can be enhanced or repressed, in an analog way with the potentiation and depression states describing the synaptic plasticity ${ }^{25}$. 

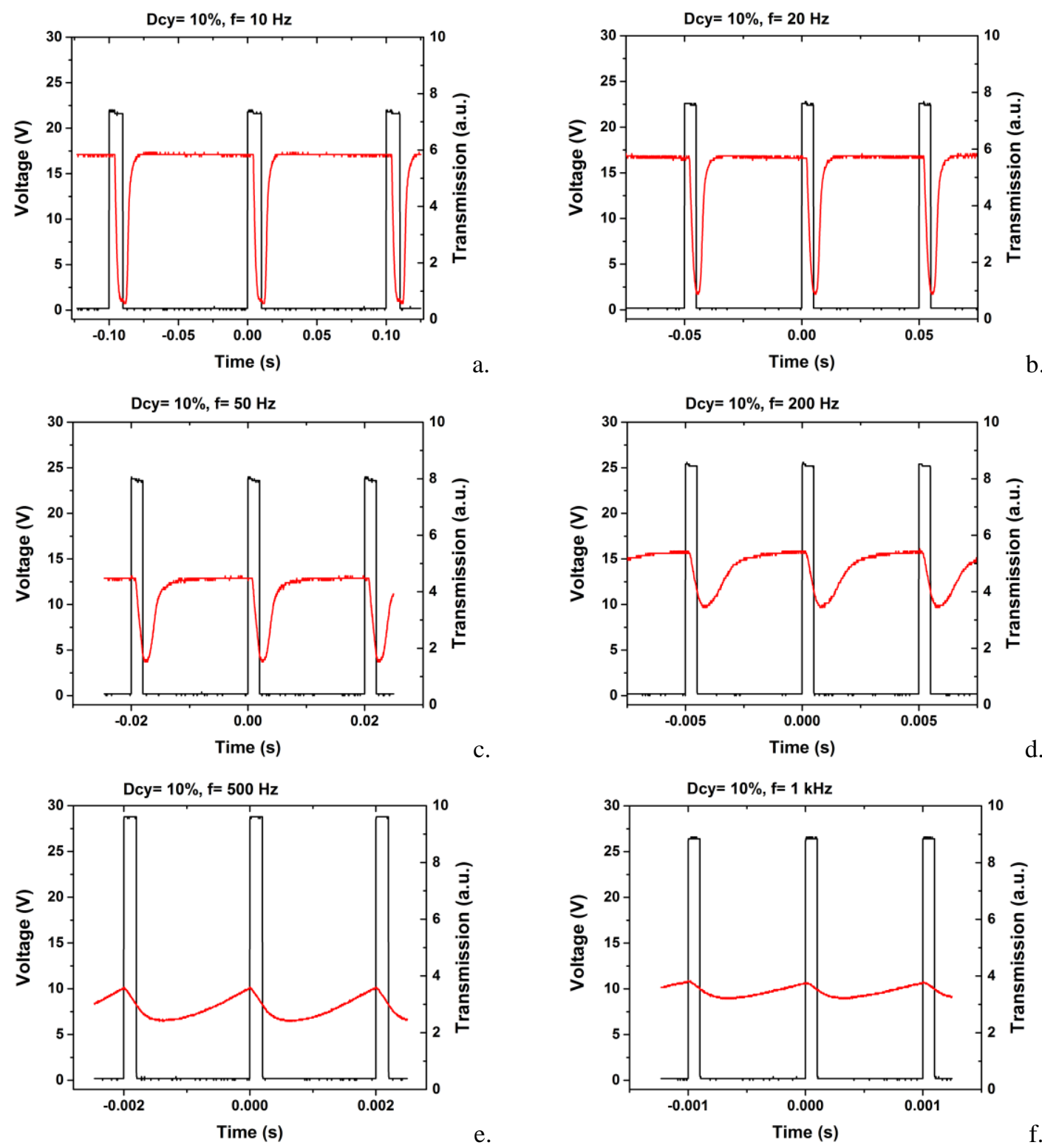

Figure 12. Frequency- dependent optical modulation variation of the $\mathrm{VO}_{2}$-based device when applying electrical pulses for a fixed DCy $=10 \%$ ( black curves: applied voltage pulses, red curves: optical transmitted power).

\section{CONCLUSIONS}

We presented the growth, structural, electrical and optical characterization of $\mathrm{VO}_{2}$ layers obtained on different types of substrates. The $\mathrm{VO}_{2}$ films obtained on sapphire substrates are mono-oriented and present electrical resistivity changes of up to five orders of magnitude across their metal- insulator transition. The temperature- and electrically triggered MIT is accompanied also by important and sharp variations in the optical characteristics of the layers. The vanadium dioxide material and the fabricated electrical-optical devices based on $\mathrm{VO}_{2}$ patterns are systems with extremely interesting nonlinear properties, both in the electrical and the optical domains. The metal-insulator transition and the associated phases percolation in the vicinity of the $\mathrm{T}_{\mathrm{MIT}}$ allows the effective modulation of optical signals with reasonable high optical modulation frequencies and the emergence of new, fascinating phenomena such as negative differential resistance and negative differential optical emittance. These remarkable properties can find interesting applications like optical modulators and limiting devices, nano- oscillators, camouflage or counterfeiting coatings, optical tunable metamaterials or bio-inspired, neuromorphic electro-optics memories. 


\section{REFERENCES}

[1] Morin, F "Oxide which shows a metal-to-insulator transition at the high temperature", Phys. Rev. Lett. 3, 34 (1959).

[2] Zylbersztejn, A and Mott, M.F., "Metal-insulator transition in vanadium dioxide", Phys. Rev. B 11, 4383 (1975).

[3] Berlung, C. N. and Guggenheim, H. "Electronic Properties of VO2 near the Semiconductor-Metal Transition", Phys. Rev. 185, 1022 (1969).

[4] Wentzcovich, R. M., Schulz, W.W. and Allen, P. B. "VO2: Peierls or Mott-Hubbard? A view from Band Theory", Phys. Rev. Lett., 72, 3389 (1994).

[5] Imada, M., Fujimori A. and Tokura, Y. "Metal-insulator transitions", Rev. Mod. Phys. 70, 1039 (1998).

[6] Yang, Z., Ko, C. and Ramanathan, S., “Oxide Electronics Utilizing Ultrafast Metal-Insulator Transitions”, Annu. Rev. Mater. Res. 41, 337 (2011).

[7] Stefanovich, G., Pergament, A. and Stefanovich, D. "Electrical switching and Mott transition in VO2”, J. Phys.: Condens. Matter, 12, 8837-8845, (2000).

[8] Kim, H. T., Chae, B. G., Youn, D. H., Maeng, S. L., Kim, G., Kang, K. Y., and Lim, Y. S. "Mechanism and observation of Mott transition in VO2-based two- and three-terminal devices", New J. Phys., 6, 52 (2004).

[9] Cavalleri, A., Tóth, Cs., Siders, C.W., Squier, J. A., Ráksi, F., Forget, P. and Kieffer, J. C. "Femtosecond Structural Dynamics in VO2 during an Ultrafast Solid-Solid Phase Transition", Physical Review Letters, 87(23) 237401-4, (2001).

[10] Li, G., X. Wang, J. Liang, A. Ji, M. Hu, F. Yang, J. Liu, N. Wu, H. Chen, H. 2nd IEEE International Nanoelectronics Conference (INEC 2008), 921-923(2008).

[11] Strelcov, E., Y. Lilach, A. Kolmakov, “Gas Sensor Based on Metal- Insulator Transition in VO2 Nanowire Thermistor”, Nano Letters 9, 2322$2326(2009)$

[12] Qazilbash, M.M., M. Brehm, B.G. Chae, P.C. Ho, G.O. Andreev, B.J. Kim, S.J. Yun, A.V. Balatsky, M.B. Maple, F. Keilmann, H.T. Kim, D.N Basov, "Mott transition in VO2 revealed by infrared spectroscopy and nano-imaging", Science 318, 1750 (2007).

[13] Givernaud, J., A. Crunteanu, J.C. Orlianges, A. Pothier, C. Champeaux, A. Catherinot, P. Blondy, "Microwave power limiting devices based on the semiconductor metal transition in Vanadium dioxide thin films", IEEE Transactions on Microwave Theory and Techniques, 58 (9), 2352$2361(2010)$

[14] Liu, M., H. Y. Hwang, H. Tao, A. C. Strikwerda, K. Fan, G. R. Keiser, A. J. Sternbach, K. G. West, S. Kittiwatanakul, J. Lu, S. A. Wolf, F. G. Omenetto, X. Zhang, K. A. Nelson, R. D. Averitt, "Terahertz-field-induced insulator-to-metal transition in vanadium dioxide metamaterial", Nature, 487, 345 (2012).

[15] Garry, G., O. Durand, A. Lorderereau, "Structural, electrical and optical properties of pulsed laser deposited VO2 thin films on R- and C-sapphire planes", Thin Solid Films 453-454, 427 (2004).

[16] Ruzmetov, D. Z., Kevin T. N., Venkatesh Ramanathan, S. "Structure-functional property relationships in rf-sputtered vanadium dioxide thin films", Journal of Applied Physics 102, 113715 (2007).

[17] Nihei, Y., Y. Sasakawa, K. Okimura, “Advantages of Inductively Coupled Plasma -Assisted Sputtering for Preparation of Stoichiometric VO2 films with Metal-Insulator Transition”, Thin Solid Films, 516, 3572-3576 (2008).

[18] Leroy, J, A. Bessaudou, F. Cosset, A. Crunteanu, "Structural, electrical and optical properties of thermochromic VO2 thin films obtained by reactive electron beam evaporation", Thin Solid Films 520, pp. 4823-4825, (2012)

[19] Crunteanu, A., J. Givernaud, J. Leroy, David Mardivirin, C. Champeaux, J.-C. Orlianges, A. Catherinot and P. Blondy, "Voltage- and currentactivated metal-insulator transition in VO2-based electrical switches: a lifetime operation analysis", Sci. Technol. Adv. Mater. 11, 065002 (2010).

[20] Aetukuri, Nagaphani B. Gray, Alexander X. Drouard, Marc Cossale, Matteo Gao, Li Reid, Alexander H. Kukreja, Roopali Ohldag, Hendrik Jenkins, Catherine A. Arenholz, Elke Roche, Kevin P. Dürr, Hermann A. Samant, Mahesh G. Parkin, Stuart S. P. "Control of the metal-insulator transition in vanadium dioxide by modifying orbital occupancy”, Nature Physics 9, 661-666 (2013).

[21] Kats, Mikhail A. Sharma, Deepika Lin, Jiao Genevet, Patrice Blanchard, Romain Yang, Zheng Qazilbash, M. Mumtaz Basov, D. N. Ramanathan, Shriram Capasso, Federico, "Ultra-thin perfect absorber employing a tunable phase change material”, Appl. Phys. Lett.101, 221101 (2012).

[22] Kats, Mikhail A.,Blanchard, Romain, Zhang, Shuyan, Genevet, Patrice, Ko, Changhyun, Ramanathan, Shriram, Capasso, Federico, "Vanadium Dioxide as a Natural Disordered Metamaterial: Perfect Thermal Emission and Large Broadband Negative Differential Thermal Emittance", Phys. Rev. X 3, 041004 (2013).

[23] J. Leroy, A. Crunteanu, A. Bessaudou, F. Cosset, C. Champeaux, and J.-C. Orlianges, "High-speed metal-insulator transition in vanadium dioxide films induced by an electrical pulsed voltage over nano-gap electrodes", Appl. Phys. Lett. 100, 213507 (2012).

[24] Beaumont, A., J. Leroy, J.-C. Orlianges, A. Crunteanu, "Current-induced electrical self-oscillations across out-of-plane threshold switches based on VO2 layers integrated in crossbars geometry", Journal of Applied Physics 115, 154502 (2014).

[25] Jo,S.H., Ting Chang, Idongesit Ebong, Bhavitavya B. Bhadviya, Pinaki Mazumder, and Wei Lu, "Nanoscale Memristor Device as Synapse inNeuromorphic Systems", Nano Lett. 10, 1297-1301 (2010). 